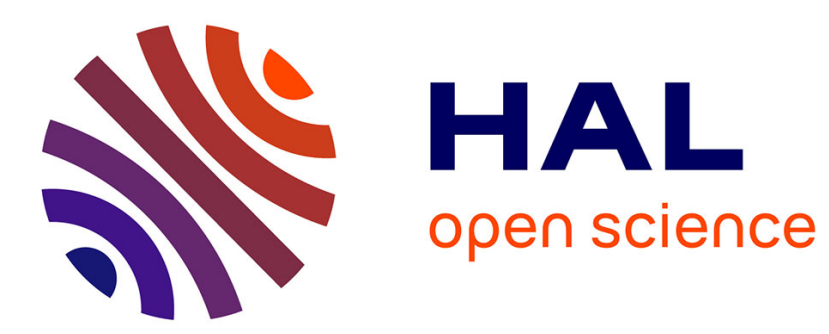

\title{
Study on color spaces for single image enrolment face authentication
}

Baptiste Hemery, Jean-Jacques Schwartzmann, Christophe Rosenberger

\section{To cite this version:}

Baptiste Hemery, Jean-Jacques Schwartzmann, Christophe Rosenberger. Study on color spaces for single image enrolment face authentication. IAPR International Conference on Pattern Recognition (ICPR), 2010, France. 10.1109/ICPR.2010.311 . hal-00990881

\section{HAL Id: hal-00990881 https://hal.science/hal-00990881}

Submitted on 14 May 2014

HAL is a multi-disciplinary open access archive for the deposit and dissemination of scientific research documents, whether they are published or not. The documents may come from teaching and research institutions in France or abroad, or from public or private research centers.
L'archive ouverte pluridisciplinaire HAL, est destinée au dépôt et à la diffusion de documents scientifiques de niveau recherche, publiés ou non, émanant des établissements d'enseignement et de recherche français ou étrangers, des laboratoires publics ou privés. 


\title{
Study on Color Spaces for Single Image Enrolment Face Authentication
}

\author{
B. Hemery ${ }^{1}$, J.J. Schwartzmann ${ }^{2}$, C. Rosenberger ${ }^{1}$ \\ ${ }^{1}$ GREYC Laboratory, ENSICAEN-Université de Caen-CNRS \\ 6 boulevard Maréchal Juin, 14000 Caen, France \\ \{bhemery, christophe.rosenberger\}@greyc.ensicaen.fr \\ 2 Orange Labs, 14000 Caen, France \\ jeanjacques.schwartzmann@orange-ftgroup.com
}

\begin{abstract}
We propose in this paper to study different color spaces for representing an image for the face authentication application. We used a generic algorithm based on a matching of keypoints using sift descriptors computed on one color component. Ten color spaces have been studied on four large and significant benchmark databases (ENSIB, FACES94, AR and FERET). We show that all color spaces do not provide the same efficiency and the use of the color information allows an interesting improvement of verification results.
\end{abstract}

\section{Introduction}

Biometric technologies are now present in our daily life environment. Among all the existing biometric modalities, 2D face authentication and identification remains a great challenge $[1,10]$. Nowadays, all images captured by classical devices such as webcams, mobile phones or video monitoring cameras are colored ones and are represented in the RGB color space. Nevertheless, many proposed face recognition methods in the literature process only gray-scale images (only use of the luminance information). One classical approach to develop a color face verification system is to consider it as a multimodal system where the verification scores for the three color components are fused. The objective of this paper is to answer many questions within this context: Which gain can we expect using the color information instead of using only the luminance ? Is there one or multiple color spaces for the representation of the image in order to obtain better performances for face verification? Is it interesting to fuse different color spaces considering the performance and the computation time ? This paper is organized as follows. Section two describes previous works in the literature concerning the study in color spaces for face recognition. In section three, we detail the proposed method using a generic face verification system and 10 different color spaces. Section four illustrates the experimental results on four large face benchmarks. We conclude and give some perspectives of this work in section five.

\section{Previous works}

We can find in the literature some studies on color spaces for face recognition. One of the first work on this topic has been done in 1999 by Torres et al. [11]. This work was done on a very small database composed of 120 images on four color spaces where none significant difference had been noticed between them. Yoo et al. [13] made a comparative study on color spaces for face recognition. Their system used multiple images for the enrolment and EigenFace as training algorithm. They put into obviousness that the efficiency of the color spaces were dependent of the face alterations (expression, illumination...). The color space $\mathrm{YCbCr}$ was identified as an interesting color one for this application. Sadeghi et al. [9] proposed an interesting study on color spaces for face verification. Multiple color spaces have been studied and fused for the verification process. Authors proposed a method to automatically defining the set of color spaces to use for the verification process. Yand and Liu derived in 2008 a discriminant color space from the RGB one to increase the performance [12]. They obtained verification results $10 \%$ better than using the RGB color space. We can notice that none of these studies has been realized on a 
single image enrolment context. This kind of approach does not require any learning and highlights the efficiency of a color space. Moreover, generally only one benchmark database has been used, it is so difficult to say if these results can be generalized in all cases. In order to contribute to solve this problem, we propose a new study.

\section{Developed method}

For this study, we use a generic face biometric system based on the computation of the well known SIFT descriptors (simplified version of the system published in [8]). As pre-processing, histogram equalization is used to normalize the registered face photometrically [9]. The computation of the face pattern is described in Figure 1, it is used both for the enrolment and verification steps.

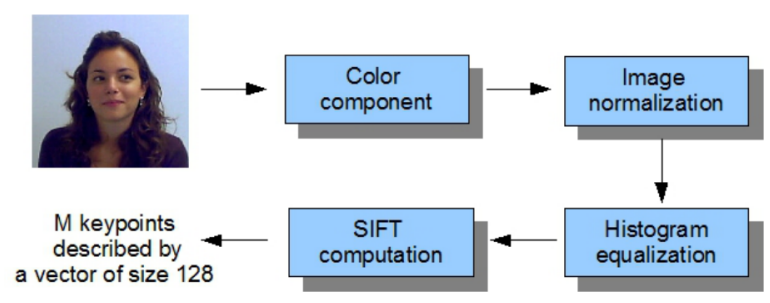

Figure 1. Computation of the pattern

In order to verify the identity of an individual (in the context of a single enrolment biometric system), we have to compute a similarity measure between its template and a new capture. We used a method based on the double associations of the two sets of detected keypoints proposed in [8]. In this case, the number of associations (matched keypoints) corresponds to the similarity of the two faces. The processing of color images for face verification can be solved by a multimodal approach consisting in merging the verification scores for different color components (see Figure 2). We used as normalization method the tanh algorithm as it provides better results [3]. The sum of the normalized scores is used as fusion function because it is simple and generally efficient. In this paper, we have studied 10 color spaces: GRAY (luminance), RGB (Red, Green, Blue), HSV (Hue, Saturation, Value), YCbCr (luma, blue and red chroma), HSL (Hue, Saturation, Luminance), OTHA (color space for segmentation), YIQ (NTSC standard), CMY (Cyan, Magenta, Yellow), CIE XYZ (human color perception) and CIE La*b* (derivative of CIE XYZ).

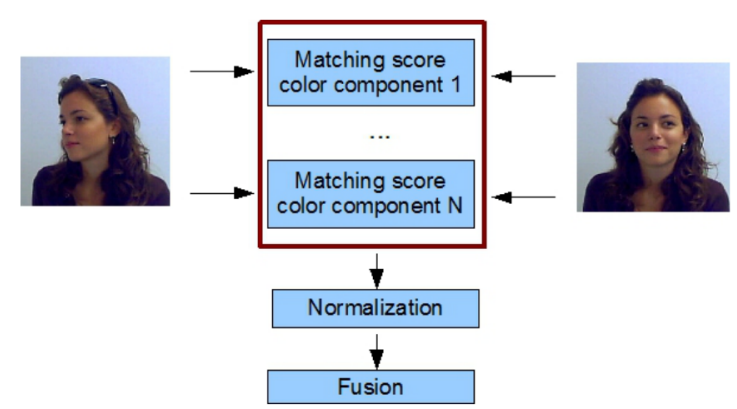

Figure 2. Color face processing

\section{Experimental results}

We detail in this section the experimental protocol and the results we obtained. For the performance evaluation process, we used four significant benchmark databases of different difficulties with many artifacts (illumination, pose variation, occlusion):

1. FACES94 Database [5]: This database is composed of 152 individuals and 20 samples per individual. These images have been captured in regulated illumination and the variation of expression is moderated (see Figure 3). The first sample is used as reference.

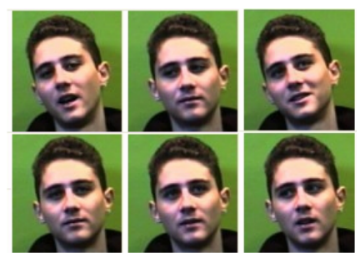

Figure 3. Samples from FACES94

2. FERET Database [7, 6]: It is composed of 725 individuals with from 5 to 91 samples per individual (the average value is 11). Each sample corresponds to a pose angle, illumination and expression. The first sample is used as reference.
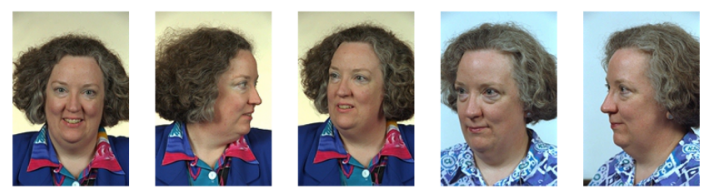

Figure 4. Samples from FERET 
3. AR Database [4]: It is composed of 120 individuals and 26 samples per individual. These included images captured under different conditions of illumination, expression and occlusion. The first sample is used as reference.

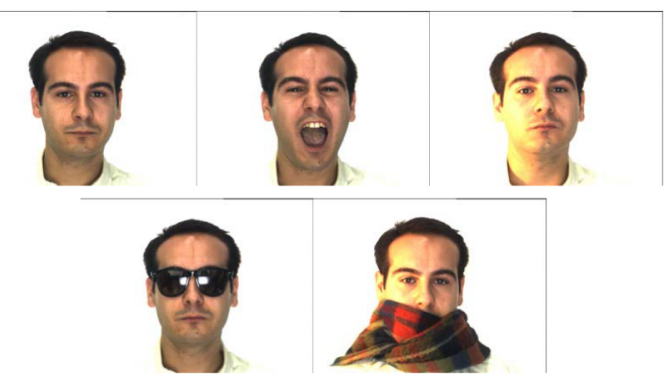

Figure 5. Samples from AR

4. ENSIB Database [2]: It is composed of 100 individuals and 40 samples per individual. Each sample corresponds to one pose from the left one to the right. The frontal image is used as reference.

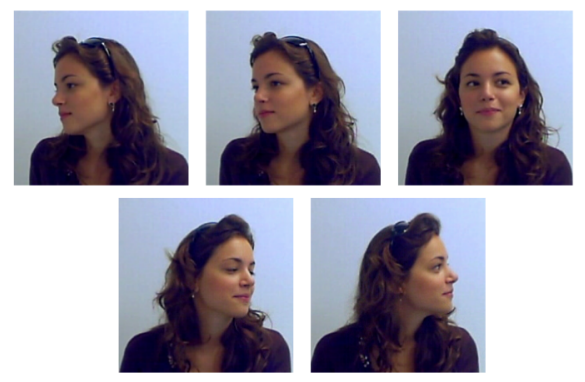

Figure 6. Samples from ENSIB

We apply the generic biometric system described in Figure 1 on one color component. This comparative study has aim to determine the color components (i.e. transformation) that provide the best performance for face verification. Results have to be considered relatively to each others. We describe the performance of one color component based on the EER value (Equal Error Rate). It corresponds to the behavior of a biometric system for the value of the decision threshold set as a compromise between the FAR (False Acceptance Rate) and the FRR one (False Rejection Rate). We use a single image for the enrolment, that means no learning is realized (the reliability of the color space is not biased). Table 1 gives the performance of each color component for the four benchmark databases. We can see first that the performance of the generic system

\begin{tabular}{|c|c|c|c|c|c|}
\hline Space & $\mathrm{CC}$ & FACES94 & ENSIB & AR & FERET \\
\hline GRAY & & 0.10 & 11.6 & 13.7 & 21.8 \\
\hline \multirow{3}{*}{ RGB } & $\mathrm{R}$ & 0.27 & 12.5 & 8.7 & 19 \\
\hline & $\mathrm{G}$ & 0.23 & 10.7 & 8.3 & 20.3 \\
\hline & B & 0.27 & 14.4 & 8.7 & 18.6 \\
\hline \multirow{3}{*}{ HSV } & $\mathrm{H}$ & 7.6 & 24.9 & 26.7 & 19.4 \\
\hline & $\mathrm{S}$ & 3.7 & 16.9 & 13.1 & 16.5 \\
\hline & V & 0.17 & 11.2 & 8 & 27.3 \\
\hline \multirow{3}{*}{$\mathrm{YCbCr}$} & $\mathrm{Y}$ & 0.15 & 11.7 & 6.9 & 26.9 \\
\hline & $\mathrm{Cb}$ & 1.5 & 15.3 & 12.8 & 12.1 \\
\hline & $\mathrm{Cr}$ & 1 & 17.4 & 17.8 & 11.6 \\
\hline \multirow{3}{*}{ YIQ } & $\mathrm{Y}$ & 0.13 & 6.6 & 6.6 & 27.4 \\
\hline & $\mathrm{I}$ & 1.8 & 18.6 & 13.9 & 15.6 \\
\hline & Q & 2.8 & 26.7 & 20.3 & 19.4 \\
\hline \multirow{3}{*}{ HSL } & $\mathrm{H}$ & 7.3 & 24.9 & 26.8 & 20.9 \\
\hline & $\mathrm{S}$ & 4.4 & 14.7 & 17.7 & 17.8 \\
\hline & $\mathrm{L}$ & 0.15 & 10 & 6.7 & 28.5 \\
\hline \multirow{3}{*}{ OTHA } & I1 & 0.15 & 10 & 6.7 & 28.5 \\
\hline & I2 & 1.2 & 18.1 & 14 & 15.9 \\
\hline & I3 & 1.5 & 26.2 & 18.3 & 17.8 \\
\hline \multirow{3}{*}{ CMY } & $\mathrm{C}$ & 0.11 & 10.2 & 8.7 & 27.6 \\
\hline & $\mathrm{M}$ & 0.18 & 10 & 8.1 & 19.8 \\
\hline & $\mathrm{Y}$ & 0.23 & 10 & 6.3 & 27.3 \\
\hline \multirow{3}{*}{ XYZ } & $\mathrm{X}$ & 0.16 & 11 & 5.5 & 28.9 \\
\hline & Y & 0.19 & 11.7 & 6.6 & 27.9 \\
\hline & $\mathrm{Z}$ & 0.25 & 10.6 & 6.7 & 27.6 \\
\hline \multirow{3}{*}{$\mathrm{La}^{*} \mathrm{~b}^{*}$} & $\mathrm{~L}$ & 0.13 & 9.1 & 9 & 27.4 \\
\hline & $a^{*}$ & 2.7 & 18.4 & 19.2 & 19.3 \\
\hline & $\mathrm{b}^{*}$ & 3.6 & 15.9 & 13.2 & 17.4 \\
\hline
\end{tabular}

Table 1. Performance evaluation of each color component: value of the EER in \%

is different for these four benchmarks meaning some databases are easier to process (as for example, the same algorithm gives a performance 200 times better for FACES94 than for FERET). Second, the behavior of the different color components is relatively different for all databases (we put in bold the three better results for each database). Results given by the different color components for FACES94 and ENSIB are similar, this is due to the fact that no illumination alteration is present for these databases (which may influence the color component efficiency). Third, we can see that in most cases, many color components give better results than the luminance information (GRAY raw). These results show that no color component provides the best results in all the cases, the use of the four databases was really necessary. 


\begin{tabular}{|l|cccc|}
\hline Color space & FACES94 & ENSIB & AR & FERET \\
\hline GRAY & $\mathbf{0 . 1 0}$ & 11.6 & 13.7 & 21.8 \\
\hline RGB & 0.18 & 9.6 & $\mathbf{4 . 6}$ & $\mathbf{1 1 . 3}$ \\
\hline HSV & 0.30 & 11.5 & 9.5 & 14.5 \\
\hline YCbCr & 0.15 & 10.3 & 7.8 & 11.8 \\
\hline YIQ & 0.17 & 9.6 & 7.9 & 14.3 \\
\hline HSI & $\mathbf{0 . 1 1}$ & 9.9 & 6.4 & 16.2 \\
\hline OTHA & 0.17 & 9.6 & 7.5 & 14.6 \\
\hline CMY & 0.19 & 8.6 & $\mathbf{4 . 4}$ & 19.3 \\
\hline XYZ & 0.15 & 9 & 5.6 & 21 \\
\hline La*b* & $\mathbf{0 . 1 1}$ & $\mathbf{8 . 4}$ & 8.1 & 15.5 \\
\hline TOTAL & 0.12 & $\mathbf{7 . 2}$ & $\mathbf{4 . 4}$ & $\mathbf{9 . 3}$ \\
\hline
\end{tabular}

\section{Acknowledgments}

Portions of the research in this paper use the FERET database of facial images collected under the FERET program, sponsored by the DOD Counterdrug Technology Development Program Office.

\section{References}

[1] T.-W. Chen, S.-C. Hsu, and S.-Y. Chien. Automatic feature-based face scoring in surveillance systems. In ISM '07: Proceedings of the Ninth IEEE International Symposium on Multimedia, pages 139-146, Washington, DC, USA, 2007. IEEE Computer Society.

Table 2. Performance evaluation using color information: value of the EER in \%

We fusion each component among a color space using the process described in Figure 2. Table 2 gives the performance we obtain by taking into account the color information. When none illumination alteration is present in the database (FACES94 and ENSIB), the $\mathrm{La}^{*} \mathrm{~b} *$ color space gives one of the best result. In the other cases, the RGB color space is a good candidate. We can notice for the most difficult database (FERET) the benefit to take into account the color information as it permits to divide by 2 the EER value compared to the GRAY one. The "TOTAL" row corresponds to the fusion of all color spaces. For the Faces94 database, this fusion does not improve the performance (all the EER values were already low). In all other cases, we obtain the best results but the difference is not significant compared to the use of the best color space as the computation time is multiplied by ten.

\section{Conclusion and perspectives}

We presented in this paper a study on color spaces for face authentication. We showed on four significant face databases that the ten studied color spaces have different behaviors. This conclusion was made possible because we used four different databases which is one of our main contribution. We put into obviousness the benefit of the CIE La*b* and RGB color spaces for this application. The benefit of the color information was also illustrated.

We intend to work in the future on the definition of color spaces best suited for face verification especially in terms of performance and robustness.

[2] B. Hemery, C. Rosenberger, and H. Laurent. The ensib database : a benchmark for face recognition. In International Symposium on Signal Processing and its Applications (ISSPA), special session "Performance Evaluation and Benchmarking of Image and Video Processing”, 2007.

[3] A. Jain, K. Nandakumar, and A. Ross. Score normalization in multimodal biometric systems. Pattern Recognition, 12(38):2270-2285, 2005.

[4] A. Martinez and R. Benavente. The ar face database. CVC Tech. Report, 24, 1998.

[5] U. of Essex. Faces 94 database, face recognition data, 1994.

[6] P. Phillips, H. Moon, S. Rizvi, and P. Rauss. The feret evaluation methodology for face recognition algorithms. IEEE Trans. Pattern Analysis and Machine Intelligence, 22:1090-1104, 2000.

[7] P. Phillips, H. Wechsler, J. Huang, and P. Rauss. The feret database and evaluation procedure for face recognition algorithms. Journal of Image and Vision Computing, 16(5):295-306, 1998.

[8] C. Rosenberger and L. Brun. Similarity-based matching for face authentication. In International Conference on Pattern Recognition (ICPR), 2008.

[9] M. T. Sadeghi, S. Khoshrou, and J. Kittler. Colour feature selection for face authentication. In IAPR Conference on Machine Vision Applications, 2007.

[10] V. Struc, R. Gajsek, and N. Pavesic. Principal gabor filters for face recognition. In IEEE Third International Conference on Biometrics: Theory, Applications and Systems (BTAS), Washington, DC, USA, 2009.

[11] L. Torres, J. Reutter, and L. Lorente. The importance of the color information in face recognition. In International Conference on Image Processing, volume 3, pages 627-631, 1999.

[12] J. Yang and C. Liu. A discriminant color space method for face representation and verification on a largescale database. In International Conference on Pattern Recognition (ICPR), 2008.

[13] S. Yoo, R.-H. Park, and D.-G. Sim. Investigation of color spaces for face recognition. In IAPR Conference on Machine Vision Applications, 2007. 